\title{
VALOR DE LA BIOPSIA VULVAR EN EL DIAGNÓSTICO DE UQUEN ESCLEROSO EN PACIENTES CON ALTERACIONES VULVARES CRÓNICAS
}

\author{
Pablo Sanhueza $R^{1,2}$, Nicole Yaksic B. ${ }^{2, a}$, Karina Chahuán $I^{1}$ \\ ${ }^{1}$ Facultad de Ciencias de la Salud, Universidad Andrés Bello. ${ }^{2}$ Unidad de Ginecología, Municipalidad de Santiago.
}

a Matrona.

\section{RESUMEN}

Se presenta un estudio prospectivo donde se realizó biopsia vulvar a pacientes con síntomas y/o alteraciones vulvares crónicas de más de un año de evolución. Se destaca la importancia de la biopsia vulvar como un procedimiento sencillo, barato y exento de complicaciones, vital en la confirmación diagnóstica de liquen escleroso.

\section{PALABRAS CLAVES: Liquen escleroso, vulva, biopsia}

\section{SUMMARY}

A prospective study is presented. A vulvar biopsy was carried out in patients with symptoms and/or chronic vulvar alterations of more than one year of evolution. We stands out the importance of the vulvar biopsy like a simple, cheap procedure and exempt of complications, vital in the diagnostic confirmation of lichen sclerosus.

\section{KEY WORDS: Lichen sclerosus, vulva, biopsy}

\section{INTRODUCCIÓN}

Liquen escleroso es una enfermedad inflamatoria crónica cutánea benigna de origen desconocido, que puede afectar áreas genitales y no genitales en ambos sexos, siendo más frecuente su localización ano genital con un $85 \%$ a $98 \%$ de los casos (1).

Típicamente se presenta en pacientes con o sin signos clínicos, que presentan síntomas vulvares crónicos, de varios meses e incluso años de evolución y en las cuales se han intentado tratamientos tópicos o sistémicos sin mayores resultados.

Su localización vulvar está caracterizada por síntomas como prurito intenso, dolor, sensación urente e irritación, acompañado generalmente de lesiones vulvares con eritema, descamación, lesiones por grataje, erosiones y fisuras, más frecuentemente ubicadas en los surcos labiales y en la región perianal. Puede acompañarse de disuria, dificultad para defecar, dispareunia y se puede asociar a vitiligo de la región genital; en algunos pacientes puede cursar en forma asintomática (1, $2,3,4,5)$.

Se estima que afecta a menos del $1 \%$ de las mujeres, con una relación de mujer a hombre de 10:1. Se encuentra en todos los grupos etáreos, siendo más frecuente en la infancia y en la postmenopausia. No existen factores de riesgos hasta ahora identificados, y no se relaciona con el uso de anticonceptivos o terapias de reemplazo 
hormonal $(1,2,4)$. Si bien su origen es hasta ahora desconocido, existen numerosos estudios que intentan definir una etiología, dentro de las que destacan su probable origen autoinmune, genético, viral e infeccioso $(6,7,8,9)$.

Es conocida la relación entre carcinoma de la vulva con liquen escleroso y neoplasias vulvares intraepiteliales de alto grado. Sin embargo, el real potencial maligno de estas alteraciones es incierto. Existen estudios donde se ha demostrado que el riesgo de carcinoma de células escamosas de la vulva en mujeres con liquen escleroso es de 4 a $5 \%$. A la vez, los cambios histológicos compatibles con liquen escleroso se asocian a carcinoma de células escamosas de la vulva entre 28 a $76 \%$ de los casos (10,11, 12, 13, 14, 15, 16).

El tratamiento de las pacientes con liquen escleroso es difícil. Actualmente existen varias opciones, entre las que destacan tratamientos tópicos con testosterona $2 \%$, clobetasol dipropionato $0,05 \%$, mometasona furoato $0,1 \%$, tacrolimus $0,1 \%$, tratamientos sistémicos con retinoides y tratamientos quirúrgicos con vaporización con láser de dióxido de carbono, denervación vulvar, criocirugía e incluso vulvectomía simple $(1,2,4,5,17,18,19$, 20, 21).

El objetivo de este estudio es evaluar el valor de la biopsia vulvar en el diagnóstico de liquen escleroso en pacientes con síntomas vulvares crónicos.

\section{MATERIAL Y MÉTODOS}

Se realizó un estudio prospectivo a corto plazo donde se diseñó un protocolo para tomar una biopsia vulvar en aquellas pacientes de cualquier edad que tuvieran síntomas o alteraciones vulvares de más de un año de evolución, con antecedentes de haber sido tratadas previamente con medidas empíricas sin resultados satisfactorios, y una vez descartadas otras patologías como sospecha de lesiones cancerosas o infecciosas.

En un período de 15 meses, se atendieron 4.480 pacientes ginecológicas, en la Unidad de Ginecología de la llustre Municipalidad de Santiago y en el consultorio privado de uno de los autores (PSR). Del total de pacientes, 5 presentaban síntomas vulvares crónicos de más de 1 año de evolución con variados tratamientos sin resultados positivos. Las cinco pacientes eran postmenopáusicas y el promedio de edad fue de 64 años. Los síntomas y signos presentes fueron prurito intenso (5 pacientes), eritema (4 pacientes), dispareunia (3 pacientes), erosión vulvar (3 pacientes), borramiento de pliegues y acartonamiento de la piel en la región genital (1 paciente), asociación con vitiligo genital (1 paciente) (Figura 1). En una paciente el único síntoma era el prurito intenso refractario a tratamiento y no presentaba alteraciones vulvares visibles.

Biopsia vulvar. Previa aseptización con povidona yodada se realizó una biopsia simple en la consulta con hoja de bisturí y anestésico local en la zona de mayor sospecha de aproximadamente $1 \mathrm{~cm}$ de diámetro, la que en los cinco casos con alteraciones vulvares correspondió al lado externo del labio menor de la vulva. Se suturó con seda $3 / 0$ con puntos separados. No se emplearon antibióticos profilácticos. Las pacientes fueron controladas a los 10 (extracción de puntos) y 30 días de efectuada la biopsia.

\section{RESULTADOS}

De las 5 pacientes en las que se realizó la biopsia vulvar, en 4 se confirmó histológicamente el diagnóstico de liquen escleroso vulvar (Figura 2). La paciente restante presentó una neoplasia intraepitelial vulvar (VIN) de bajo grado sin presencia de liquen. No se presentaron complicaciones como sangrados, hematomas o infecciones, y ninguna paciente experimentó dolor durante la toma de la biopsia o posterior a ella. El resultado estético fue bueno, no existiendo cicatrices o indicios de la biopsia en el control realizado a los 30 días.

\section{DISCUSIÓN}

Liquen escleroso vulvar es una patología extremadamente poco frecuente. El infrecuente diag-

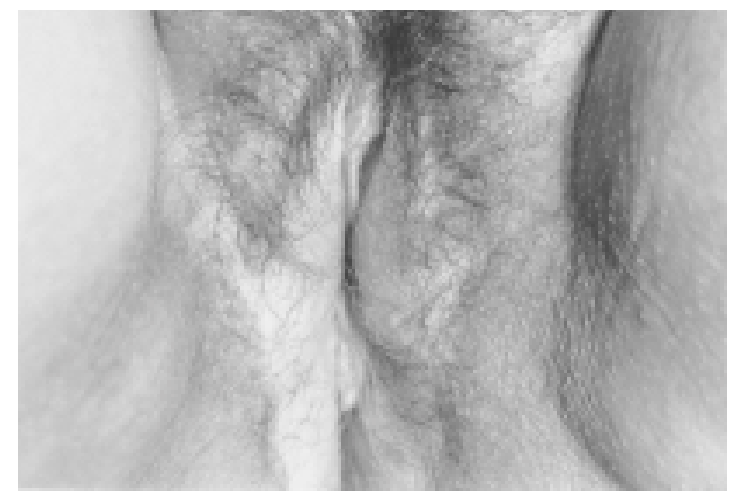

Figura 1. Paciente de 67 años con lesiones por liquen escleroso vulvar asociado a vitiligo. Se aprecia eritema, borramiento de los pliegues vulvares y acartonamiento de la piel. 


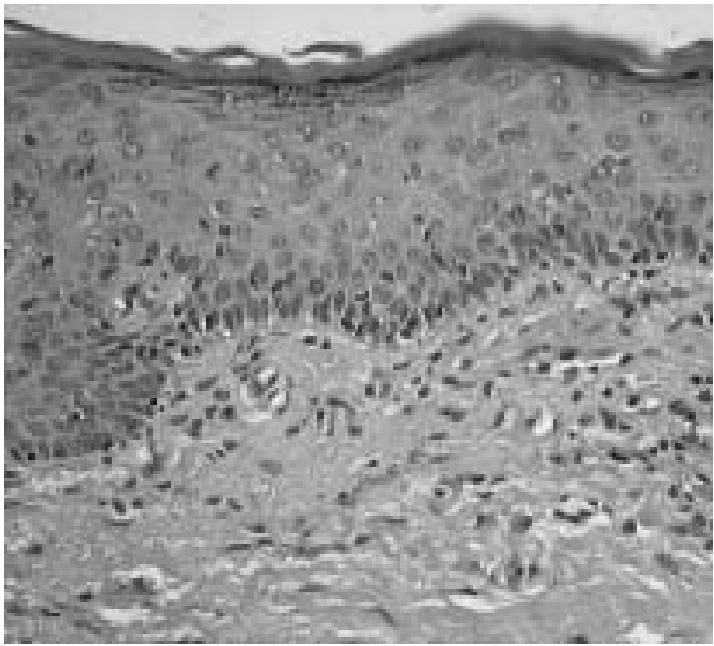

Figura 2. Corte histológico de piel de región vulvar de uno de los casos del estudio visto en microscopía óptica con tinción hematoxilina-eosina, aumento $40 \times 12$, que evidencia aplanamiento de las crestas interpapilares, fibrosis de la dermis, inflamación y edema del estrato basal.

nóstico está dado por la baja incidencia y por la escasa sospecha clínica y confirmación histológica. Generalmente se trata de pacientes postmenopáusicas con antecedentes de alteraciones y síntomas vulvares de varios meses y años de evolución, caracterizadas principalmente por prurito intenso, dispareunia, eritema y erosiones vulvares, pudiendo incluso presentarse en forma asintomática. En nuestro medio no existen estudios de prevalencia. El diagnóstico se basa en la sospecha clínica que debe ser confirmada histológicamente.

En este estudio se demuestra que frente a la sospecha clínica de liquen escleroso u otras alteraciones vulvares, la realización de una biopsia vulvar en el consultorio es un procedimiento rápido, sencillo, económico, prácticamente sin complicaciones, con buenos resultados estéticos y funcionales, con alto rendimiento diagnóstico frente a la sospecha clínica.

La confirmación histológica nos permite iniciar un tratamiento adecuado y descartar una lesión maligna de la vulva. En este último sentido debe destacarse que las pacientes con liquen escleroso vulvar tienen mayor riesgo de presentar carcinoma de la vulva, riesgo que se ha estimado entre 4 a $5 \%$. Es por esta razón que las pacientes con diagnóstico de liquen escleroso vulvar deben tener controles frecuentes y en caso de sospecha deben ser repetidas las biopsias cuantas veces se considere necesario, no existiendo actualmente una recomendación de la frecuencia de las tomas o en qué características clínicas o histológicas del liquen escleroso sea necesaria su repetición.

Con respecto al tratamiento existen varias modalidades terapéuticas. Como primer enfoque existen regímenes tópicos de variados compuestos. Dentro de estos destaca clobetasol dipropionato al $0,05 \%$ el cual es significativamente mejor que la aplicación de testosterona propionato al $2 \%$, demostrando una resolución de los síntomas en el $75 \%$ de los casos de mujeres que usaron clobetasol comparado con sólo el $25 \%$ de las mujeres que usaron testosterona, mejorando no sólo desde el punto de vista clínico, si no también desde el punto de vista histológico, esto último, sólo presentado en el grupo con clobetasol. Además, la testosterona puede producir efectos adversos, principalmente hiperandrogenismo, lo que puede ocurrir en el $30 \%$ de las pacientes, a diferencia del $5 \%$ de reacciones indeseables reportado para el grupo tratado con clobetasol. Los régimenes tópicos descritos para el clobetasol dipropionato al 0,05\% son dos veces al día por un mes, luego una vez al día por el siguiente mes y posteriormente 2 veces a la semana por 3 meses $(2,18,22)$.

Recientemente, dos nuevas opciones terapéuticas han demostrado favorables resultados: tacrolimus al $0,1 \%$, un potente inmunosupresor usado ampliamente en la prevención de rechazo de trasplante hepático, aplicado en forma tópica dos veces al día por períodos prolongados, ha sido indicado en pacientes con pobre respuesta a los tratamientos estándar, no reportándose efectos adversos y presentando un excelente período de remisión de la enfermedad una vez suspendido $(1,21)$; mometasona furoato al $0,1 \%$ aplicada en forma tópica una vez al día por 4 semanas y luego 2 veces a la semana por 8 semanas parece ser una buena alternativa a los regímenes estándar de clobetasol, ofreciendo eficacia y mejoría clínica, evitando los efectos adversos al aplicar esteroides tópicos potentes tales como este último (19). Otras opciones terapéuticas incluyen vaporización con láser de dióxido de carbono y en casos más severos y resistentes a tratamiento se ha planteado la realización de una vulvectomía simple $(4,17,20)$.

\section{CONCLUSIÓN}

La biopsia vulvar en pacientes con síntomas y signos locales crónicos, permite certificar el diagnóstico de liquen escleroso y descartar patología neoplásica maligna de la vulva. 


\section{BIBLIOGRAFÍA}

1. Kunstfeld R, Kirnbauer R, Stingl G, Karlhofer FM. Successful treatment of vulvar lichen sclerosus with topical tacrolimus. Arch Dermatol 2003; 139: 85052.

2. Lorenz B, Kaufman RH, Kutzner SK. Lichen sclerosus. Therapy with clobetasol propionate. J Reprod Med 1998; 43: 790-94.

3. Fischer G, Spurrett B, Fischer A. The chronically symptomatic vulva: aetiology and management. $\mathrm{Br} \mathrm{J}$ Obstet Gynaecol 1995; 102: 773-79.

4. Powell JJ, Wojnarowska F. Lichen sclerosus. Lancet 1999; 353: 1777-83.

5. Larrabee R, Kylander DJ. Benign vulvar disorders. Identifyng features, practical management of nonneoplastic conditions and tumors. Postgraduate Medicine 2001; 109: 151-64.

6. Marren P, Yell J, Charnock M, Wojnarowska F. The association between lichen sclerosus and antigens of the HLA system. Br J Dermatol 1995; 132: 197203.

7. Goolamali SK, Barnes EW, Irvine WJ, Shuster S. Organ specific antibodies in patient with lichen sclerosus. BMJ 1974; 6: 78-9.

8. Drut R, Gómez M, Drut R, Lojo M. Human papilloma virus is present in some cases of childhood penile lichen sclerosus: an in situ hybridization and SPPCR study. Pediatr Dermatol 1998; 15: 85-90.

9. Scrimin F, Rustja S, Radillo O, Volpe C, Abrami R, Guaschino S. Vulvar lichen sclerosus: an immunologic study. Obstet Gynecol 2000; 95: 147-50.

10. Carli P, De Magnis A, Mannone F, Botti E, Taddei G, Cattaneo A. Vulvar carcinoma associated with lichen sclerosus. J Reprod Med 2003; 48: 313-18.

11. Gomez-Rueda N, Garcia A, Vighi S, Beladi M, Cardinal L, di Paola G. Epithelial alterations adjacent to invasive squamous carcinoma of the vulva. J Reprod Med 1994; 39: 526-30.

12. Leibowitch M, Neill S, Pelisse M, Meyal-Baracco M. The epithelial changes associated with squamous cell carcinoma of the vulva: A review of the clinical, histological and viral findings in 78 women. $\mathrm{Br} \mathrm{J}$
Obstet Gyanecol 1990; 97: 1135-39.

13. Vilmer C, Cavelier-Balloy B, Noguees C, Trassard M, Le Doussal V. Analysis of alterations adjacent to invasive vulvar carcinoma and their relationship with the associated carcinoma: A study of 67 cases. Eur J Gynecol Oncol 1998; 19: 25-31.

14. Saravanamuthu J, Reid W, St George D, Crow J, Rolfe K, MacLean A, y cols. The role of angiogenesis in vulvar cancer, vulvar intraepithelial neoplasia, and vulvar lichen sclerosus as determined by microvessel density analysis. Gynecologic Oncology 2003; 89: 251-58.

15. MacLean AB. Precursors of vulval cancers. Curr Obstet Gynaecol 1993; 3: 149-56.

16. Carlson JA, Ambros R, Malfetano J, Ross J, Grabowski R, Lamb P, y cols. Vulvar lichen sclerosus and squamous cell carcinoma: a cohort, case control and investigational study with historical perspective; implications for chronic inflammation and sclerosis in the development of neoplasia. Hum Pathol 1998; 29: 932-48.

17. Sinha P, Sorinola O, Luesley D. Lichen sclerosus of the vulva. Long-term steroid maintenance therapy. J Reprod Med 1999; 44: 621-24.

18. Bornstein J, Heifetz S, Kellner Y, Stolar Z, Abramovici H. Clobetasol dipropionate 0,05\% versus testosterone propionate $2 \%$ topical application for severe vulvar lichen sclerosus. Am J Gynecol 1998; 178: 80-4.

19. Cattaneo A, De Magnis A, Botti E, Sonni L, Carli P, Taddei G. Topical mometasone furoate for vulvar lichen sclerosus. J Reprod Med 2003; 48: 444-48.

20. Kartamaa M, Reitamao S. Treatment of lichen sclerosus with carbon dioxide laser varopization. $\mathrm{Br}$ J Dermatol 1997; 136: 356-59.

21. Böhm M, Frieling U, Luger TA, Bonsmann G. Successful treatment of anogenital lichen sclerosus with topical tacrolimus. Arch Dermatol 2003; 139: 922-24.

22. Bracco GL, Carli P, Sonni L, Maestrini G, De Marco A, Taddei G, y cols. Clinical and histologic effects of topical treatments of vulval lichen sclerosus - a critical evaluation. J Reprod Med 1993; 38: 37-40. 